\title{
The immune microenvironment of breast ductal carcinoma in situ
}

\author{
Elizabeth Thompson ${ }^{1}$, Janis M Taube ${ }^{1,2}$, Hillary Elwood ${ }^{3}$, Rajni Sharma ${ }^{1}$, Alan Meeker ${ }^{1}$, \\ Hind Nassar Warzecha ${ }^{4}$, Pedram Argani ${ }^{1,5}$, Ashley Cimino-Mathews ${ }^{1,5}$ and \\ Leisha A Emens ${ }^{5}$
}

${ }^{1}$ Department of Pathology, The Johns Hopkins Hospital, Baltimore, MD, USA; ${ }^{2}$ Department of Pathology and Dermatology, The Johns Hopkins Hospital, Baltimore, MD, USA; ${ }^{3}$ Department of Pathology, University of New Mexico, Albuquerque, NM, USA; ${ }^{4}$ Department of Pathology, University of Tuebingen, Tuebingen, BadenWurttenberg, Germany and ${ }^{5}$ Department of Oncology, Sidney Kimmel Cancer Center, The Johns Hopkins Hospital, Baltimore, MD, USA

\begin{abstract}
The host immune response has a key role in breast cancer progression and response to therapy. However, relative to primary invasive breast cancers, the immune milieu of breast ductal carcinoma in situ (DCIS) is less understood. Here, we profile tumor infiltrating lymphocytes and expression of the immune checkpoint ligand programmed death ligand 1 (PD-L1) in 27 cases of DCIS with known estrogen receptor (ER), progesterone receptor, and human epidermal growth factor 2 (HER-2) expression using tissue microarrays. Twenty-four cases were pure DCIS and three had associated invasive ductal carcinoma. Tumors were stained by immunohistochemistry for PD-L1, as well as the lymphocyte markers CD3, CD4, CD8, FoxP3, and CD20. The expression of PD-L1 by DCIS carcinoma cells and tumor infiltrating lymphocytes was determined, and the average tumor infiltrating lymphocytes per high power field were manually scored. None of the DCIS cells expressed PD-L1, but $81 \%$ of DCIS lesions contained PD-L1+ tumor infiltrating lymphocytes. DCIS with moderate-diffuse tumor infiltrating lymphocytes was more likely to have PD-L1+ tumor infiltrating lymphocytes $(P=0.004)$. Tumor infiltrating lymphocytes with high levels of PD-L1 expression ( $>50 \%$ cells) were seen only in triple-negative DCIS $(P=0.0008)$, and PD-L1 - tumor infiltrating lymphocytes were seen only in ER+/HER-2 $-\mathrm{DCIS}(P=0.12)$. The presence of PD-L1+ tumor infiltrating lymphocytes was associated with a younger mean patient age $(P=0.01)$. Further characterization of the DCIS immune microenvironment may identify useful targets for immune-based therapy and breast cancer prevention.

Modern Pathology (2016) 29, 249-258; doi:10.1038/modpathol.2015.158; published online 15 January 2016
\end{abstract}

Data from both laboratory studies and clinical trials support an important role for the host immune response in breast cancer progression and in patient response to therapy. ${ }^{1,2}$ Tumor infiltrating lymphocytes, both within tumor cell nests and in tumor-associated stroma, are associated with improved patient outcomes. In treatment naive triple-negative breast carcinomas, tumor infiltrating

Correspondence: Dr A Cimino-Mathews, MD, Departments of Pathology and Oncology, Johns Hopkins School of Medicine, The Sidney Kimmel Comprehensive Cancer Center at Johns Hopkins, Weinberg Building 2242, 401N, Broadway Street, Baltimore, MD 21287, USA or Dr LA Emens, MD, PhD, Department of Oncology, Johns Hopkins University School of Medicine, The Sidney Kimmel Comprehensive Cancer Center at Johns Hopkins, Bunting-Blaustein Cancer Research Building, 1650 Orleans Street, Room 409, Baltimore, MD 21231-1000, USA.

E-mail: acimino1@jhmi.edu or emensle@jhmi.edu

Received 1 September 2015; revised 7 December 2015; accepted 9 December 2015; published online 15 January 2016 lymphocytes are an independent prognostic factor for improved survival, ${ }^{3,4}$ decreased distant recurrence, ${ }^{4,5}$ and increased time to metastasis. ${ }^{6}$ The presence of tumor infiltrating lymphocytes in triple-negative breast carcinomas post-neoadjuvant therapy is also prognostic, predicting longer metastasis-free and overall survival. ${ }^{3}$ In addition, the presence of tumor infiltrating lymphocytes predicts response to therapy, as larger numbers of tumor infiltrating lymphocytes correlate with better responses to chemotherapy in triple-negative breast carcinomas and estrogen receptor (ER)-negative breast cancer, ${ }^{1,2}$ and to trastuzumab-based chemotherapy in human epidermal growth factor receptor-2 (HER-2)-positive breast carcinomas. ${ }^{5}$ Multiple tumor infiltrating lymphocyte subsets, including $\mathrm{CD}^{+}$cytotoxic $\mathrm{T}$ cells, $\mathrm{CD}^{+}$helper $\mathrm{T}$ cells, and $\mathrm{CD}^{+} \mathrm{O}^{+} \mathrm{B}$ cells, predict patient survival across the breast carcinoma subtypes. ${ }^{1,2}$ In contrast, high numbers of $\mathrm{FoxP}^{+}$regulatory $\mathrm{T}$ cells (Tregs) 
relative to $\mathrm{CD}^{+} \mathrm{T}$ cells predict decreased progression-free survival and overall survival ${ }^{7}$ in breast carcinoma patients.

Gene expression profiling defined five major molecular subtypes of invasive primary breast carcinomas with unique clinicopathologic characteristics and outcomes: luminal A, luminal B, HER-2-enriched (HER-2 ${ }^{+}$), basal-like, and normal breast-like. ${ }^{8}$ These molecular subtypes have immunohistochemical correlates, where luminal A cancers are $\mathrm{ER}^{+}$ progesterone receptor $(\mathrm{PR})^{+} \mathrm{HER}-2^{-} \mathrm{Ki} 67^{\text {low }}$, luminal $\mathrm{B}$ cancers are $\mathrm{ER}^{+} \mathrm{PR}^{+} \mathrm{HER}-2^{-} \mathrm{Ki} 7^{\text {high }}$ or $\mathrm{ER}^{+} \mathrm{PR}^{+} \mathrm{HER}-2^{+}$, HER $-2^{+}$cancers are ER ${ }^{-} \mathrm{PR}^{-} \mathrm{HER}-2^{+}$, and basal-like carcinomas are typically ER $^{-} \mathrm{PR}^{-} \mathrm{HER}-\mathrm{2}^{-}$(triple-negative breast carcinoma) with cytokeratin (CK)5/6 or epidermal growth factor (EGFR) expression. ${ }^{9}$ The luminal A phenotype has an improved prognosis relative to luminal B, HER- ${ }^{+}$, or basal-like carcinomas. ${ }^{9}$ These same gene expression profiles and immunohistochemical phenotypes are observed in ductal carcinoma in situ (DCIS), the only established precursor to invasive primary breast carcinomas. ${ }^{10}$

Although HER-2 ${ }^{+}$breast cancers and triple-negative breast carcinomas have a poor prognosis relative to other subtypes, they typically have more vigorous tumor lymphocyte infiltrates. Larger numbers of tumor infiltrating lymphocytes are seen in $\mathrm{ER}^{-}$relative to $\mathrm{ER}^{+}$carcinomas. ${ }^{11}$ In addition, genetic correlates of the immune response predict better survival in $\mathrm{ER}^{-}$and HER-2 ${ }^{+}$carcinomas, but not $\mathrm{ER}^{+}$carcinomas. $^{12}$ Although robust immune infiltrates are associated with ER $^{-}$and HER-2 ${ }^{+}$ carcinomas, evidence suggests functional skewing toward a pro-tumorigenic phenotype. High levels of Treg are more common in $\mathrm{ER}^{-}$carcinomas, ${ }^{13}$ and predict shorter progression-free and overall survival. ${ }^{7}$ In addition, relative to luminal A cancers, triple-negative breast carcinomas tend to harbor tumor infiltrating lymphocytes with the $\mathrm{T}$ helper type 2 phenotype thought to promote tumor growth. ${ }^{14}$

Growing evidence supports a major role for immune checkpoint pathways, such as those mediated by interactions between programmed cell death protein 1 (PD-1) and programmed death ligand 1 (PD-L1), in regulating antitumor responses. ${ }^{1,2}$ PD-1 (B7-1) is upregulated following the activation of lymphocytes and belongs to the B7-CD28 family. Its primary ligands are PD-L1 and PD-L2, which are inducible by IFN-: and other inflammatory cytokines on the surface of tumor cells and tumor infiltrating $\mathrm{T}$ cells, $\mathrm{B}$ cells, macrophages, and dendritic cells. ${ }^{15}$ The interaction of PD-1 with PD-L1/2 inhibits T-cell activation and proliferation. ${ }^{16}$ PD-L1 may also be constitutively expressed on tumor cells as a result of oncogenic signaling or epithelial-to-mesenchymal transition. ${ }^{17,18}$ Tumor cells may also counter an active antitumor immune response by upregulating PD-L1 expression through a process known as adaptive immune resistance. ${ }^{19}$ PD-L1 is expressed on the tumor cells of invasive breast carcinomas and their associated tumor infiltrating lymphocytes ${ }^{17,18,20}$ and correlates with lack of ER expression, increased numbers of tumor infiltrating lymphocytes, response to chemotherapy, and the triple-negative phenotype. ${ }^{21}$ The role of PD-L1 expression as a prognostic factor in breast carcinoma remains unclear. In one study, PD-L1 expression was associated with improved clinical outcomes; ${ }^{11}$ however, a separate study reported an association with negative clinical outcomes. ${ }^{22}$

We previously reported differential patterns of tumor infiltrating lymphocytes across matched primary and metastatic breast carcinomas, ${ }^{23}$ and in invasive primary breast carcinomas with associated DCIS. ${ }^{24}$ Our data suggest that tumor immunobiology evolves as tumors progress. Little is known about the immune milieu of DCIS or how the antitumor immune response evolves as breast tumors progress from in situ to invasive and then metastatic lesions. Here, we profile the composition of tumor infiltrating lymphocytes and PD-L1 expression in 27 cases of DCIS with known ER, PR, and HER-2 expression.

\section{Materials and methods}

\section{Case Selection and Tissue Microarray Construction}

This study was approved by the Institutional Review Board of the Johns Hopkins Medical Institutions. We evaluated tissue microarrays previously constructed from archived, paraffin-embedded blocks of primary DCIS, ${ }^{25,26}$ with 27 cases evaluable. Each DCIS case was sampled with 2-5 cores/tumor, with each core measuring $1.4 \mathrm{~mm}$ in diameter; 1 core/case sampled benign breast lobules. Clinicopathologic characteristics were recorded, including patient age, gender, race, tumor size, presence or absence of associated invasive carcinoma, DCIS nuclear grade, presence or absence of necrosis, ER, PR and HER-2 status, and patient outcome (local recurrence, metastasis, and survival). Carcinoma recurrence was defined as any in situ or invasive carcinoma recurrence in the ipsilateral breast, axilla, or chest wall. Younger women were defined as $\leq 40$ years old, and older women as $>40$ years old.

\section{Immunohistochemistry and Tumor Infiltrating Lymphocyte Quantification}

To characterize the DCIS subtype, tissue microarrays were stained by immunohistochemistry for ER (clone 6F11; Leica Microsystems, Bannockburn, IL, USA), PR (clone 16; Leica Microsystems), and HER-2 (Ventana 4B5; Ventana Medical Systems, Tucson, AZ, USA) using standard automated methods. Tumors were classified as Luminal 
A $\left(\mathrm{ER}^{+} / \mathrm{PR}^{+} / \mathrm{HER}-2^{-}\right)$, Luminal B $\left(\mathrm{ER}^{+} / \mathrm{PR}^{+} / \mathrm{HER}-2^{+}\right)$, $\mathrm{HER}^{-}{ }^{+}$(ER $\left.{ }^{-} / \mathrm{PR}^{-} / \mathrm{HER}^{-} 2^{+}\right)$, and triple negative (ER ${ }^{-} / \mathrm{PR}^{-} / \mathrm{HER}-2^{-}$). HER-2 positivity was defined as $\geq 10 \%$ complete strong membranous DCIS cell labeling as per the 2012 ASCO/CAP guidelines for HER-2 immunohistochemistry.

Each case was assigned a qualitative stromal tumor infiltrating lymphocyte density score on the hematoxylin and eosin (H\&E) stained sections: 0 (no tumor infiltrating lymphocytes), 1 (mild, < $<\%$ tumor area with tumor infiltrating lymphocytes ), 2 (moderate, 5-50\% tumor area with tumor infiltrating lymphocytes), and 3 (diffuse/marked, $>50 \%$ infiltration). ${ }^{19,24,27}$ Tumor infiltrating lymphocytes were scored by two pathologists (ET and ACM) blinded to clinicopathologic characteristics. Tissue microarrays were stained for CD20 (monoclonal, clone MS/L26, catalog no. 760-2531, Ventana Medical Systems), CD3 (mouse monoclonal, clone PS1, catalog no. ORG-8982, Leica Microsystems), CD4 (rabbit monoclonal, clone SP35, catalog no. q790-4423, Ventana Medical Systems), CD8 (mouse monoclonal, clone C8/C8144B, catalog no. 760-4250, Cell Marque, Rockin, CA, USA), and FoxP3 (mouse monoclonal, clone 236A/E7, catalog no. 14-4777-80, dilution 1:50; eBioscience, San Diego, CA, USA) to characterize tumor infiltrating lymphocytes as previously described. ${ }^{23}$ CD20, CD3, CD4, and CD8 expression was defined by membranous lymphocyte labeling, and FoxP3 expression was defined by nuclear labeling in lymphocytes. The total number of tumor infiltrating lymphocytes/high power field was manually counted in one high power field/tissue microarray tumor core, and averaged across the case to give the mean number of tumor infiltrating lymphocytes/high power field. Each high power field was chosen as representative of the overall tumor lymphocytic infiltration in each tumor core.

Tissue microarrays were stained for PD-L1 (B7-H1) using the murine anti-human PD-L1 antibody, clone $5 \mathrm{H} 1(2 \mu \mathrm{g} / \mathrm{ml})$ with a paired isotype murine IgG1 control as previously described. ${ }^{19}$ PD-L1 staining was scored by two pathologists (ET and ACM) blinded to patient clinicopathologic characteristics; discrepancies were adjudicated by a third pathologist (JT). PD-L1 staining on carcinoma cells and tumor infiltrating lymphocytes was scored by percent membranous staining. PD-L1 positivity by DCIS carcinoma cells was defined as $>5 \%$ membranous staining. PD-L1 positivity in tumor infiltrating lymphocytes was scored as none (0), focal $(1+;<5 \%)$, moderate $(2+; 5-50 \%)$, or marked $(3+; 51-100 \%)$ percentage of tumor infiltrating lymphocytes expressing PD-L1. PD-L1 expression was also scored as low $(\leq 50 \%$ tumor infiltrating lymphocytes) or high ( $>50 \%$ of tumor infiltrating lymphocytes).

\section{Statistical Analysis}

Statistical analysis was performed using Fisher's exact test and paired, two-tailed Student's T-test.

\section{Results}

\section{Clinicopathologic Features of DCIS Cases}

The clinicopathologic features of 27 evaluable patients with DCIS are detailed in Table 1. The median patient age was 38 years (mean 41 years, range 18-74), with $52 \%$ white, $26 \%$ black, $7 \%$ Asian, and $4 \%$ Hispanic patients. The DCIS phenotype of 26 evaluable patients included $62 \%$ luminal A, $15 \%$ luminal B, 12\% HER-2 $^{+}$, and $12 \%$ triple negative, for a total of $77 \% \mathrm{ER}^{+}$and $23 \%$ ER $^{-}$cases. The ER/PR/HER-2 status of one case could not be determined, and the case was not included in analyses that subdivided by DCIS phenotype. Most cases were nuclear grade $2(52 \%)$ or grade $3(44 \%)$. The average tumor size was $2.4 \mathrm{~cm} ; 37 \%$ were multifocal. Twenty-four cases were pure DCIS (pathologic stage pTis), without any associated invasive carcinoma. Three cases had associated infiltrating ductal carcinoma, which were also present on the tissue microarray cores. Two of these cases were triple-negative DCIS and one was luminal A DCIS. The mean and median follow-up time were 82 months and 80 months (approximately 7 years), respectively. Two (7\%) DCIS patients had an ipsilateral recurrence, both of which were nuclear grade 3; one patient with triple-negative DCIS and concurrent infiltrating ductal carcinoma developed recurrent infiltrating ductal carcinoma in the ipsilateral chest wall 1 year after initial diagnosis, and one patient with HER-2 ${ }^{+}$DCIS experienced recurrent ipsilateral DCIS 3 years after initial diagnosis. No patients received neoadjuvant therapy. All patients received adjuvant therapy for pure DCIS or infiltrating carcinoma as per the standard of care.

\section{Quantification of Tumor Infiltrating Lymphocytes in DCIS}

We first evaluated tumor infiltrating lymphocytes associated with DCIS by histopathologic density scoring on a scale from 0 to 3 . All cases had tumor infiltrating lymphocytes, with $78 \%$ showing either moderate (score 2) or diffuse (score 3) tumor infiltrating lymphocyte density (Table 2). Only two cases $(7 \%)$ contained diffuse tumor infiltrating lymphocytes. $\mathrm{ER}^{+}$DCIS and DCIS in older patients tended to have lower tumor infiltrating lymphocyte density scores than $\mathrm{ER}^{-}$DCIS or DCIS in younger patients $(P=0.28$ and 0.32 , respectively). There was only one case of grade 1 DCIS, precluding group wide comparison, and there was no discernible difference in tumor lymphocytic infiltration between the grade 2 DCIS and grade 3 DCIS. 
Table 1 Clinicopathologic characteristics of ductal carcinoma in situ cases

\begin{tabular}{|c|c|c|c|c|c|}
\hline & \multirow{2}{*}{ DCIS (all cases) } & \multicolumn{4}{|c|}{ Phenotype ${ }^{\mathrm{a}}$} \\
\hline & & Luminal $A$ & Luminal $B$ & HER-2 & Triple negative \\
\hline Total number & $n=27$ & $16(62 \%)$ & $4(15 \%)$ & $3(12 \%)$ & $3(12 \%)$ \\
\hline \multicolumn{6}{|l|}{ Age (years) } \\
\hline Median (range) & $38(18-74)$ & 45 & 37 & 33 & 37 \\
\hline$\leq 40$ & $19(70 \%)$ & $10(62 \%)$ & $4(100 \%)$ & $2(67 \%)$ & $2(67 \%)$ \\
\hline$>40$ & $8(30 \%)$ & $6(38 \%)$ & 0 & $1(33 \%)$ & $1(33 \%)$ \\
\hline \multicolumn{6}{|l|}{ Race } \\
\hline White & $14(52 \%)$ & $9(57 \%)$ & $2(50 \%)$ & $2(50 \%)$ & $1(25 \%)$ \\
\hline Black & $7(26 \%)$ & $4(25 \%)$ & $0(0 \%)$ & $0(0 \%)$ & $2(50 \%)$ \\
\hline Asian & $2(7 \%)$ & $1(6 \%)$ & $1(25 \%)$ & $0(0 \%)$ & $0(0 \%)$ \\
\hline Hispanic & $1(4 \%)$ & $1(6 \%)$ & $0(0 \%)$ & $0(0 \%)$ & $0(0 \%)$ \\
\hline Other & $3(11 \%)$ & $1(6 \%)$ & $1(25 \%)$ & $1(25 \%)$ & $0(0 \%)$ \\
\hline \multicolumn{6}{|l|}{ Nuclear grade } \\
\hline 1 & $1(4 \%)$ & $1(6 \%)$ & $0(0 \%)$ & $0(0 \%)$ & $0(0 \%)$ \\
\hline 2 & $14(52 \%)$ & $11(69 \%)$ & $2(50 \%)$ & $0(0 \%)$ & $0(0 \%)$ \\
\hline 3 & $12(44 \%)$ & $4(25 \%)$ & $2(50 \%)$ & $3(100 \%)$ & $3(100 \%)$ \\
\hline \multicolumn{6}{|l|}{ Necrosis } \\
\hline No & $6(22 \%)$ & $5(31 \%)$ & $0(0 \%)$ & $0(0 \%)$ & $0(0 \%)$ \\
\hline Yes & $21(78 \%)$ & $11(69 \%)$ & $4(100 \%)$ & $3(100 \%)$ & $3(100 \%)$ \\
\hline \multicolumn{6}{|l|}{ Multifocality } \\
\hline No & $17(63 \%)$ & $11(69 \%)$ & $2(50 \%)$ & $2(67 \%)$ & $2(67 \%)$ \\
\hline Yes & $10(37 \%)$ & $5(31 \%)$ & $2(50 \%)$ & $1(33 \%)$ & $1(33 \%)$ \\
\hline \multicolumn{6}{|l|}{ Size $(\mathrm{cm})$} \\
\hline Mean & 2.4 & 1.7 & 4.2 & 4.8 & 2.8 \\
\hline$\leq 1$ & $7(26 \%)$ & $6(38 \%)$ & $1(25 \%)$ & $0(0 \%)$ & $0(0 \%)$ \\
\hline$>1$ & $9(34 \%)$ & $7(44 \%)$ & $0(0 \%)$ & $0(0 \%)$ & $1(33 \%)$ \\
\hline$>2$ & $2(7 \%)$ & $1(6 \%)$ & $0(0 \%)$ & $1(33 \%)$ & $0(0 \%)$ \\
\hline$>3$ & $2(7 \%)$ & $1(6 \%)$ & $0(0 \%)$ & $0(0 \%)$ & $1(33 \%)$ \\
\hline$>4$ & $5(19 \%)$ & $1(6 \%)$ & $3(75 \%)$ & $1(33 \%)$ & $0(0 \%)$ \\
\hline NR & $2(7 \%)$ & $0(0 \%)$ & $0(0 \%)$ & $1(33 \%)$ & $1(33 \%)$ \\
\hline \multicolumn{6}{|l|}{ Recurrence } \\
\hline No & $25(93 \%)$ & $16(100 \%)$ & $0(0 \%)$ & $2(67 \%)$ & $2(67 \%)$ \\
\hline Yes & $2(7 \%)$ & $0(0 \%)$ & $0(0 \%)$ & $1(33 \%)$ & $1(33 \%)$ \\
\hline \multicolumn{6}{|c|}{ Associated infiltrating carcinoma } \\
\hline No & $24(89 \%)$ & $15(94 \%)$ & $0(0 \%)$ & $0(0 \%)$ & $1(33 \%)$ \\
\hline Yes & $3(11 \%)$ & $1(6 \%)$ & $0(0 \%)$ & $0(0 \%)$ & $2(67 \%)$ \\
\hline
\end{tabular}

Abbreviations: cm, centimeter; DCIS, ductal carcinoma in situ; HER-2, human epidermal growth factor 2; $n$, number; NR, not reported.

${ }^{a}$ The phenotype for one case was unable to be determined.

We quantified subsets of tumor infiltrating lymphocytes using CD3, CD4, CD8, CD20, and FoxP3 staining. $\mathrm{CD}^{+} \mathrm{T}$ cells predominated across all DCIS subtypes at all ages, with slightly more $\mathrm{CD} 4^{+} \mathrm{T}$ cells than $\mathrm{CD}^{+} \mathrm{T}$ cells on average. $\mathrm{CD} 20^{+} \mathrm{B}$ cells were the next most common tumor infiltrating lymphocytes, followed by $\mathrm{FoxP}^{+}$Treg (Table 2). On average, $\mathrm{ER}^{-}$DCIS contained higher numbers of all tumor infiltrating lymphocyte subsets than $\mathrm{ER}^{+} \mathrm{DCIS}$, and $\mathrm{ER}^{+}$DCIS was more likely to have to have a high CD8/FoxP3 ratio $(\geq 4)$ than $\mathrm{ER}^{-}$DCIS. DCIS in young women also contained higher numbers of all tumor infiltrating lymphocyte subsets relative to older women; however, these differences were not statistically significant. There was no difference in tumor infiltrating lymphocyte subset distribution between the nuclear grade 2 DCIS and grade 3 DCIS.
Although the case numbers are small and results should be interpreted with caution, differences in tumor infiltrating lymphocytes were also seen in DCIS with concurrent infiltrating ductal carcinoma $(n=3)$, and in DCIS that later recurred $(n=2)$. The two cases of DCIS with diffuse tumor infiltrating lymphocytes (score 3) were DCIS with concurrent infiltrating ductal carcinoma $(P=0.009)$. DCIS with concurrent infiltrating ductal carcinoma showed higher levels of infiltrating $\mathrm{CD}^{+} \mathrm{T}$ cells and $\mathrm{CD} 20^{+} \mathrm{B}$ cells than cases with only in situ disease, but this was not statistically significant. DCIS that recurred had higher numbers of all tumor infiltrating lymphocyte subsets relative to DCIS that did not recur. DCIS that recurred also had the greatest number of $\mathrm{CD}^{+} \mathrm{T}$ cells of any subset of DCIS cases examined. However, this DCIS phenotype also showed a lower $\mathrm{CD} 8 / \mathrm{FoxP} 3$ ratio, indicating a commensurate increase in regulatory $\mathrm{T}$ cells in these two cases. 
Table 2 Immune parameters of ductal carcinoma in situ

\begin{tabular}{|c|c|c|c|c|c|c|c|c|c|c|c|c|c|c|c|c|}
\hline \multirow[b]{2}{*}{ Immune parameter } & \multicolumn{3}{|c|}{ Phenotype } & \multirow[b]{2}{*}{ P-value } & \multicolumn{2}{|c|}{ Age (years) } & \multirow[b]{2}{*}{ P-value } & \multicolumn{2}{|c|}{ Concurrent IDC } & \multirow[b]{2}{*}{$\mathrm{P}$-value } & \multicolumn{2}{|c|}{ Recurrence } & \multirow[b]{2}{*}{ P-value } & \multicolumn{2}{|c|}{ TIL PD-L1 status } & \multirow[b]{2}{*}{ P-value } \\
\hline & $\begin{array}{c}\text { All cases } \\
\mathrm{n}=27\end{array}$ & $\begin{array}{c}\text { ER positive } \\
\mathrm{n}=20\end{array}$ & $\begin{array}{c}\text { ER negative } \\
\mathrm{n}=6\end{array}$ & & $\begin{array}{c}\leq 40 \\
19(70 \%)\end{array}$ & $\begin{array}{c}>40 \\
8(30 \%)\end{array}$ & & $\begin{array}{c}\text { No } \\
24(89 \%)\end{array}$ & $\begin{array}{c}\text { Yes } \\
3(11 \%)\end{array}$ & & $\begin{array}{c}\text { No } \\
25(93 \%)\end{array}$ & $\begin{array}{c}\text { Yes } \\
2(7 \%)\end{array}$ & & $\begin{array}{c}\text { Negative } \\
5(19 \%)\end{array}$ & $\begin{array}{c}\text { Positive } \\
22(81 \%)\end{array}$ & \\
\hline \multicolumn{17}{|c|}{ Tumor infiltrating lymphocyte densityb } \\
\hline None: 0 & 0 & 0 & 0 & \multirow{5}{*}{0.28} & 0 & 0 & \multirow{5}{*}{0.32} & 0 & 0 & \multirow{5}{*}{1} & 0 & 0 & \multirow{5}{*}{1} & 0 & 0 & \\
\hline Focal: 1 & $6(22 \%)$ & $6(30 \%)$ & 0 & & $3(16 \%)$ & $3(38 \%)$ & & $6(25 \%)$ & 0 & & $6(24 \%)$ & 0 & & $4(80 \%)$ & $2(9 \%)$ & \multirow{4}{*}{$0.004^{*}$} \\
\hline Moderate: 2 & $19(70 \%)$ & $13(65 \%)$ & $5(83 \%)$ & & $15(19 \%)$ & $4(50 \%)$ & & $18(75 \%)$ & $1(33 \%)$ & & $19(76 \%)$ & $1(50 \%)$ & & $1(20 \%)$ & $18(82 \%)$ & \\
\hline Diffuse: 3 & $2(7 \%)$ & $1(5 \%)$ & $1(17 \%)$ & & $1(5 \%)$ & $1(13 \%)$ & & 0 & $2(67 \%)$ & & 0 & $1(50 \%)$ & & 0 & $2(9 \%)$ & \\
\hline $\begin{array}{l}\text { Moderate or } \\
\text { diffuse: } 2-3\end{array}$ & $21(78 \%)$ & $14(70 \%)$ & $6(100 \%)$ & & $16(84 \%)$ & $5(62 \%)$ & & $18(75 \%)$ & $3(100 \%)$ & & $19(76 \%)$ & $2(100 \%)$ & & $1(20 \%)$ & $20(91 \%)$ & \\
\hline $\mathrm{CD} 3 / \mathrm{hpf}$ & 41.5 & 37.3 & 58.2 & 0.29 & 43.6 & 36.9 & 0.71 & 43.4 & 27.3 & 0.53 & 39.7 & 59.9 & 0.77 & 18.6 & 47.3 & 0.15 \\
\hline $\mathrm{CD} 4 / \mathrm{hpf}$ & 19.3 & 17 & 30.2 & 0.22 & 21.7 & 13.7 & 0.41 & 19.4 & 18.4 & 0.94 & 18.1 & 33.8 & 0.87 & 6.7 & 22.2 & 0.18 \\
\hline $\mathrm{CD} 8 / \mathrm{hpf}$ & 16.8 & 14.3 & 25.8 & 0.12 & 19.6 & 10.3 & 0.16 & 15.5 & 27.3 & 0.23 & 15.4 & 34.5 & 0.34 & 7.4 & 18.4 & 0.1 \\
\hline Foxp3/hpf & 8.3 & 6.3 & 15.3 & 0.06 & 8.9 & 6.9 & 0.66 & 8.4 & 7 & 0.13 & 7.5 & 17.3 & 0.72 & 1.46 & 10 & 0.3 \\
\hline CD20/hpf & 12.8 & 11.2 & 19.9 & 0.49 & 13.8 & 10.3 & 0.76 & 12.1 & 18.3 & 0.7 & 10.5 & 41.5 & 0.45 & 0.67 & 15.5 & 0.1 \\
\hline CD8/FoxP3 ${ }^{\mathrm{C}}$ & $n=25$ & $n=19$ & $n=6$ & \multirow{5}{*}{0.56} & $n=17$ & $n=8$ & \multirow{5}{*}{$\begin{array}{l}0.28 \\
0.40\end{array}$} & $n=22$ & $n=3$ & \multirow{5}{*}{0.54} & $n=23$ & $n=2$ & \multirow{3}{*}{0.37} & $n=5$ & $n=20$ & \multirow{5}{*}{$\begin{array}{l}1 \\
1 \\
\text { ) } \\
1\end{array}$} \\
\hline$<1$ & $5(20 \%)$ & $3(18 \%)$ & $2(33 \%)$ & & $2(12 \%)$ & $3(38 \%)$ & & $5(23 \%)$ & 0 & & $4(17 \%)$ & $1(50 \%)$ & & $1(20 \%)$ & $4(20 \%)$ & \\
\hline$\geq 1$ & $20(80 \%)$ & $16(84 \%)$ & $4(67 \%)$ & & $15(88 \%)$ & $5(62 \%)$ & & $17(77 \%)$ & $3(100 \%)$ & & $19(83 \%)$ & $1(50 \%)$ & & $4(80 \%)$ & $16(80 \%)$ & \\
\hline$<4$ & $15(60 \%)$ & $10(53 \%)$ & $5(83 \%)$ & & $9(53 \%)$ & $6(75 \%)$ & & $14(64 \%)$ & $1(33 \%)$ & & $13(57 \%)$ & $2(100 \%)$ & 0.50 & $3(60 \%)$ & $12(60 \%)$ & \\
\hline$\geq 4$ & $10(40 \%)$ & $9(47 \%)$ & $1(17 \%)$ & & $8(47 \%)$ & $2(25 \%)$ & & $8(36 \%)$ & $2(67 \%)$ & & $10(43 \%)$ & $\begin{array}{c}2(100 / 0) \\
0\end{array}$ & & $2(40 \%)$ & $8(40 \%)$ & \\
\hline \multicolumn{17}{|l|}{ CD20/hpf } \\
\hline$<5$ & $16(59 \%)$ & $13(65 \%)$ & $2(33 \%)$ & \multirow[t]{2}{*}{0.35} & $10(52 \%)$ & $6(75 \%)$ & \multirow[t]{2}{*}{0.40} & $14(58 \%)$ & $2(67 \%)$ & \multirow[t]{2}{*}{1} & $15(63 \%)$ & 0 & \multirow[t]{2}{*}{0.118} & $5(100 \%)$ & $11(50 \%)$ & \multirow{2}{*}{0.06} \\
\hline$>5$ & $11(41 \%)$ & $7(35 \%)$ & $4(67 \%)$ & & $9(48 \%)$ & $2(25 \%)$ & & $10(42 \%)$ & $1(33 \%)$ & & $10(40 \%)$ & $2(100 \%)$ & & $0(0 \%)$ & $11(50 \%)$ & \\
\hline \multicolumn{17}{|c|}{ Tumor infiltrating lymphocyte PD-L1 status } \\
\hline PD-L1 pos & $22(81 \%)$ & $14(70 \%)$ & $6(100 \%)$ & 0.29 & $18(96 \%)$ & $4(50 \%)$ & $0.02 *$ & $19(79 \%)$ & $3(100 \%)$ & 1 & $20(80 \%)$ & $2(100 \%)$ & 1 & & & \\
\hline PD-L1 ${ }^{\text {neg }}$ & $5(19 \%)$ & $5(25 \%)$ & $0(0 \%)$ & & $1(5 \%)$ & $4(50 \%)$ & & $5(21 \%)$ & $0(0 \%)$ & & $5(20 \%)$ & $0(0 \%)$ & & & & \\
\hline
\end{tabular}

Abbreviations: DCIS, ductal carcinoma in situ; ER, estrogen receptor; hpf, high power field; IDC, invasive ductal carcinoma; lo, low; n, number; neg, negative; pos, positive; TNBC, triple-negative breast cancer.

${ }^{\mathrm{a}} P$-values were calculated using Fisher's exact test or two-tailed Student's $T$-test. Asterisks indicate statistically significant differences of $P<0.05$.

b The $P$-values for tumor infiltrating lymphocyte density are derived from comparison between the group tumor infiltrating lymphocyte density $0-1$ and group tumor infiltrating lymphocyte density $2-3$.

Only 25 patients had calculable CD8/FoxP3 ratios (ie, two cases with unevaluable FoxP3 counts were excluded) 

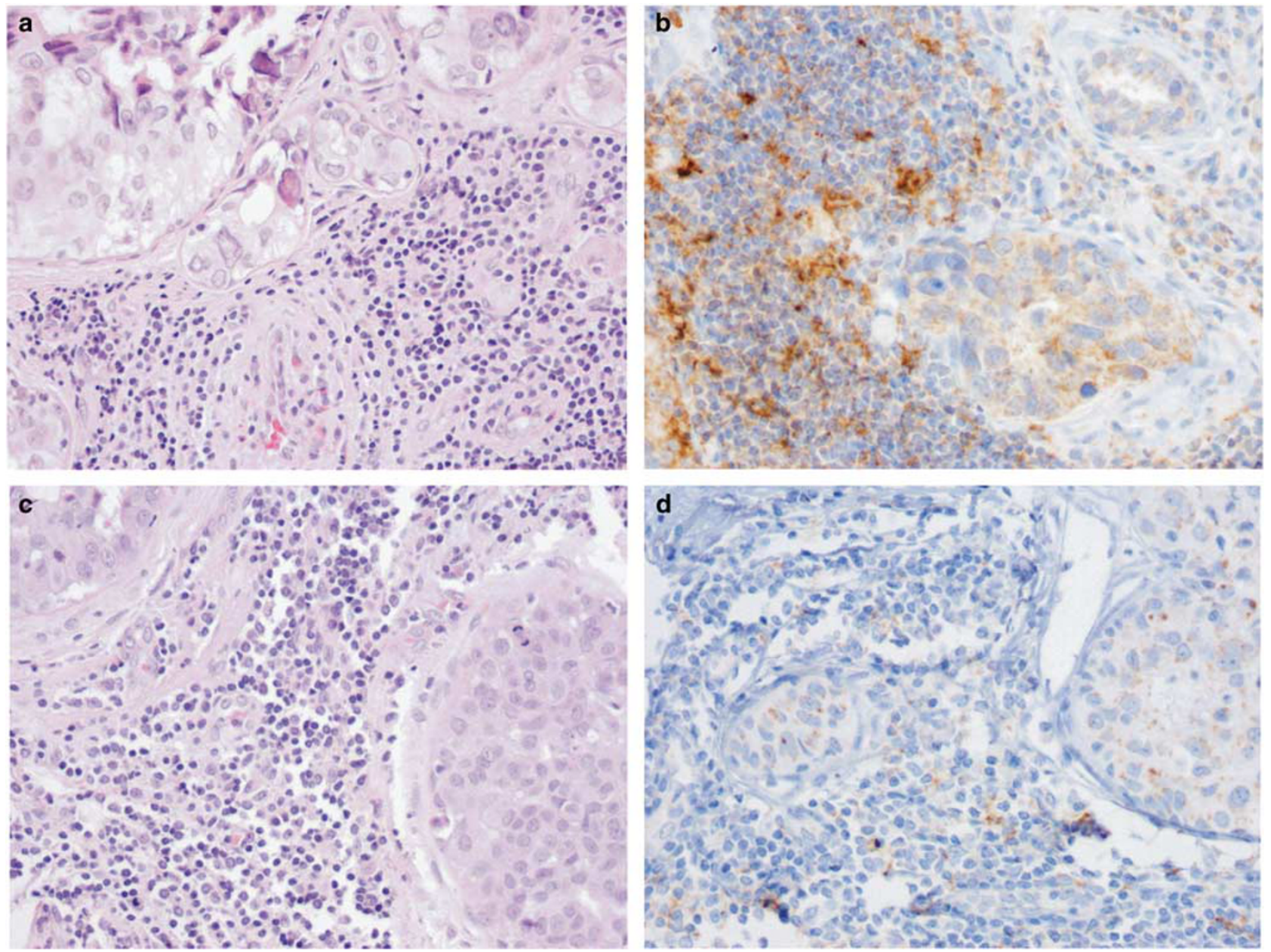

Figure 1 Programmed death ligand 1 (PD-L1) staining patterns differ in triple-negative and estrogen receptor (ER) ${ }^{+}$ductal carcinoma in situ (DCIS). All cases of triple-negative DCIS (a, hematoxylin and eosin, H\&E) contain tumor infiltrating lymphocytes with high PD-L1 ${ }^{+}$ expression ( $>50 \%$ cells) (b). ER ${ }^{+}$DCIS (c, H\&E) shows low ( $<50 \%$ cells) or absent PD-L1 tumor infiltrating lymphocyte staining (d).

\section{PD-L1 Expression in DCIS and Tumor Infiltrating Lymphocytes}

We next examined the cell surface expression of PD-L1 by DCIS tumor cells and associated tumor infiltrating lymphocytes. There was no expression of PD-L1 on DCIS tumor cells in any case, although non-specific PD-L1 staining was seen within the necrotic debris of central comedonecrosis. In DCIS with concurrent infiltrating ductal carcinoma $(n=3)$, the PD-L1 status was concordant (negative) between in situ and invasive components, as we have previously reported. ${ }^{24}$ Although DCIS tumor cells were PD-L1 ${ }^{-}, 81 \%$ of the DCIS cases showed PD-L1 expression by tumor infiltrating lymphocytes (Figure 1a and $\mathrm{b}$ and Table 2). All DCIS cases with PD-L1 ${ }^{-}$tumor infiltrating lymphocytes were of the $\mathrm{ER}^{+}$luminal A phenotype $(P=0.12)$ (Figure 1c and d), while all ER ${ }^{-}$cases had PD-L1 ${ }^{+}$tumor infiltrating lymphocytes (Table 3). PD-L1 expression was also scored as low $(\leq 50 \%$ of tumor infiltrating lymphocytes) or high ( $>50 \%$ of tumor infiltrating lymphocytes). All luminal A, luminal B, and HER-2 ${ }^{+}$
DCIS with PD-L1 ${ }^{+}$tumor infiltrating lymphocytes showed low PD-L1 expression on tumor infiltrating lymphocytes (Table 3). In contrast, $100 \%$ of triple-negative DCIS had high expression of PD-L1 on tumor infiltrating lymphocytes $(P=0.0008)$. In addition, all cases with high expression of PD-L1 on tumor infiltrating lymphocytes were nuclear grade 3 DCIS compared with nuclear grade $2 \quad(P=0.07)$ (Table 3). The average age of DCIS patients with PD-L1 ${ }^{+}$and PD-L1 ${ }^{-}$tumor infiltrating lymphocytes was 38 and 54 years $(P=0.01)$, respectively, with $80 \%$ of DCIS containing PD-L1 ${ }^{-}$tumor infiltrating lymphocytes occurred in older patients $(P=0.02)$. All high-risk DCIS (concurrent infiltrating ductal carcinoma and recurrent DCIS) had PD-L1 ${ }^{+}$tumor infiltrating lymphocytes, though this was not statistically significant.

DCIS with low tumor infiltrating lymphocyte density scores (score 1) was more likely to be $\mathrm{PD}^{-} 1^{-}$than PD-L1 ${ }^{+}(P=0.004)$. In contrast, cases of DCIS with moderate or diffuse tumor infiltrating lymphocyte density scores (scores 2-3) were more likely to be ${\mathrm{PD}-\mathrm{L} 1^{+}}^{+}$than $\mathrm{PD}-\mathrm{L1}^{-}$(Figure 2 and 
Table 3 Relationship of tumor infiltrating lymphocyte PD-L1 labeling to ductal carcinoma in situ phenotype and nuclear grade

Tumor infiltrating lymphocyte PD-L1 status

\begin{tabular}{|c|c|c|c|c|c|}
\hline & & \\
\hline & & $P D-L 1^{p o s}$ & $P D-L 1^{h i}$ a & $P D-L 1^{l o ~ b}$ & $P D-L 1^{\text {neg }}$ \\
\hline \multicolumn{6}{|l|}{ Phenotype } \\
\hline Luminal A & $n=16(62 \%)$ & $11(52 \%)$ & 0 & $11(61 \%)$ & $5(100 \%)$ \\
\hline Luminal B & $n=4(15 \%)$ & $4(19 \%)$ & 0 & $4(22 \%)$ & 0 \\
\hline Her-2 & $n=3(12 \%)$ & $3(14 \%)$ & 0 & $3(16 \%)$ & 0 \\
\hline Triple negative & $n=3(12 \%)$ & $3(14 \%)$ & $3(100 \%)$ & 0 & 0 \\
\hline Total & $n=26$ & $n=21$ & $n=3$ & $n=18$ & $n=5$ \\
\hline \multicolumn{6}{|l|}{ Nuclear grade } \\
\hline Grade 1 & $n=1(4 \%)$ & $1(5 \%)$ & 0 & $1(6 \%)$ & 0 \\
\hline Grade 2 & $n=14(52 \%)$ & $11(52 \%)$ & 0 & $11(61 \%)$ & $3(60 \%)$ \\
\hline Grade 3 & $n=12(44 \%)$ & $9(43 \%)$ & $3(100 \%)$ & $6(33 \%)$ & $2(40 \%)$ \\
\hline Total & $n=27$ & $n=22$ & $n=3$ & $n=18$ & $n=5$ \\
\hline
\end{tabular}

Abbreviations: DCIS, ductal carcinoma in situ; HER-2, human epidermal growth factor 2; hi, high; lo, low; n, number; neg, negative; yr, years old. ${ }^{\text {a PD-L1 }}{ }^{\text {hi }}$ expression was defined as $>50 \%$ tumor infiltrating lymphocytes with PD-L1 labeling.

bD-L1 ${ }^{\text {lo }}$ expression was defined as $\leq 50 \%$ TIL with PD-L1 labeling.

Table 2). DCIS with PD-L1 ${ }^{+}$tumor infiltrating lymphocytes had higher numbers of tumor infiltrating lymphocytes across all subsets examined when compared to tumors with PD-L1 ${ }^{-}$tumor infiltrating lymphocytes, though this was not statistically significant (Figure 2 and Table 2). Both PD-L1 ${ }^{+}$and PD-L1 ${ }^{-}$DCIS had similar CD8/FoxP3 ratios.

\section{Discussion}

Here, we evaluate tumor infiltrating lymphocyte composition and PD-L1 expression in breast DCIS. Our cohort is primarily pure DCIS of nuclear grade 2-3, and our data reveal several characteristics of this cohort. First, tumor infiltrating lymphocytes are present within all cases of DCIS examined. A trend toward different patterns of tumor infiltrating lymphocyte distribution and CD8/Foxp3 ratios are seen in $\mathrm{ER}^{-}$relative to $\mathrm{ER}^{+}$DCIS and younger women relative to older women with DCIS. Second, while DCIS carcinoma cells do not express cell surface PD-L1 in this study, the majority of DCIS-associated tumor infiltrating lymphocytes are PD-L1 ${ }^{+}$. The three DCIS cases with high PD-L1 expression on tumor infiltrating lymphocytes $\left(>50 \%\right.$ PD-L1 ${ }^{+}$tumor infiltrating lymphocytes) are triple negative and high nuclear grade. In three cases where DCIS was associated with concurrent infiltrating ductal carcinoma, no cell surface PD-L1 expression was seen on the carcinoma cells of either the in situ or invasive component. Although the conclusions are limited by the small number of cases with concurrent invasion, this finding is in agreement with our previous study showing concordance of carcinoma cell PD-L1 status by frank infiltrating ductal carcinoma and its associated DCIS. ${ }^{24}$ Third, PD-L1 expression by tumor infiltrating lymphocytes is associated with a younger patient age. Fourth, PD-L1 expression by tumor infiltrating lymphocytes is associated with higher overall levels of tumor infiltrating lymphocytes within DCIS. Our findings suggest an active immune response within breast DCIS and support tumor infiltrating lymphocyte expression of PD-L1 as a marker of downregulation of the body's immune response within DCIS. The presence of PD-L1 ${ }^{+}$tumor infiltrating lymphocytes with in situ breast carcinoma suggests that investigation of immune-based therapies may be warranted even in pre-invasive disease.

Most studies of the immunobiology of breast carcinomas have focused on frankly invasive primary breast carcinomas, with little attention to immune infiltrates associated with in situ lesions. Studies in mouse models of breast cancer have identified robust $\mathrm{CD}^{+}$T-cell responses associated with hyperplastic, pre-neoplastic lesions. ${ }^{28}$ Genomic analyses of DCIS and invasive breast cancers reveal activation of interleukin signaling pathway profiles, extracellular matrix pathways, and cell-cell adhesion pathways; activation of these pathways is absent or low in adjacent benign breast tissues. Furthermore, genomic profiling of DCIS shows patterns consistent with cytotoxic T-cell signaling, expression of chemokines known to recruit T cells, IFN- $\alpha$ signaling, and NOX4 activity, an oxygen-sensing NADPH oxidase related to the production of reactive oxygen species by granulocytes. ${ }^{14}$ One study reported clusters of tumor infiltrating lymphocytes adjacent to involved ducts or in the interductal stroma, often associated with plump endothelial-lined vessels suggestive of high endothelial venule-like vasculature. ${ }^{29}$ DC-based vaccination of DCIS patients also suggest that distinct DCIS phenotypes may have different immunogenicity at baseline, as vaccination produced more complete responses in $\mathrm{ER}^{-}$DCIS than in $\mathrm{ER}^{+}$DCIS. ${ }^{30,31}$

Our findings suggest that an active adaptive immune response may exist within DCIS. Nuclear grade 2-3 DCIS is associated with tumor infiltrating lymphocyte phenotypes similar to those seen in invasive breast carcinomas. This includes higher levels of tumor 

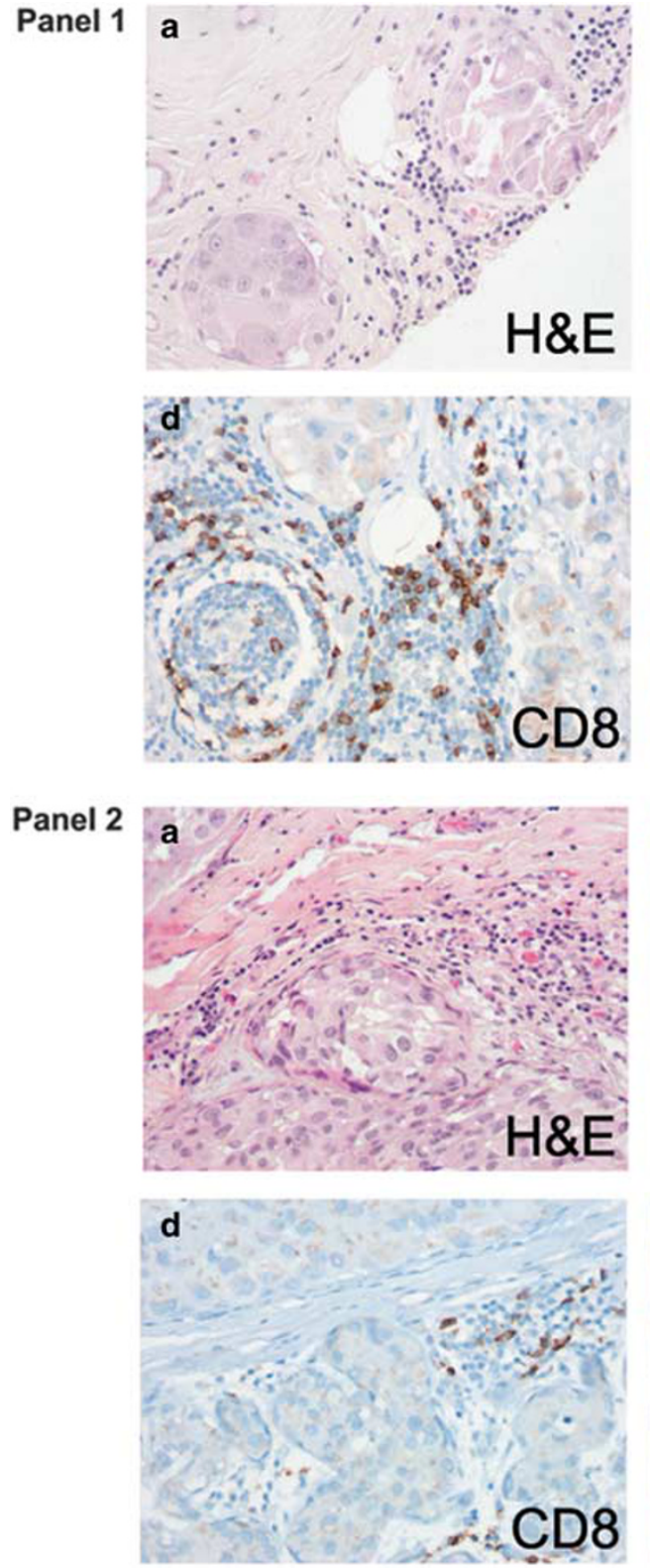
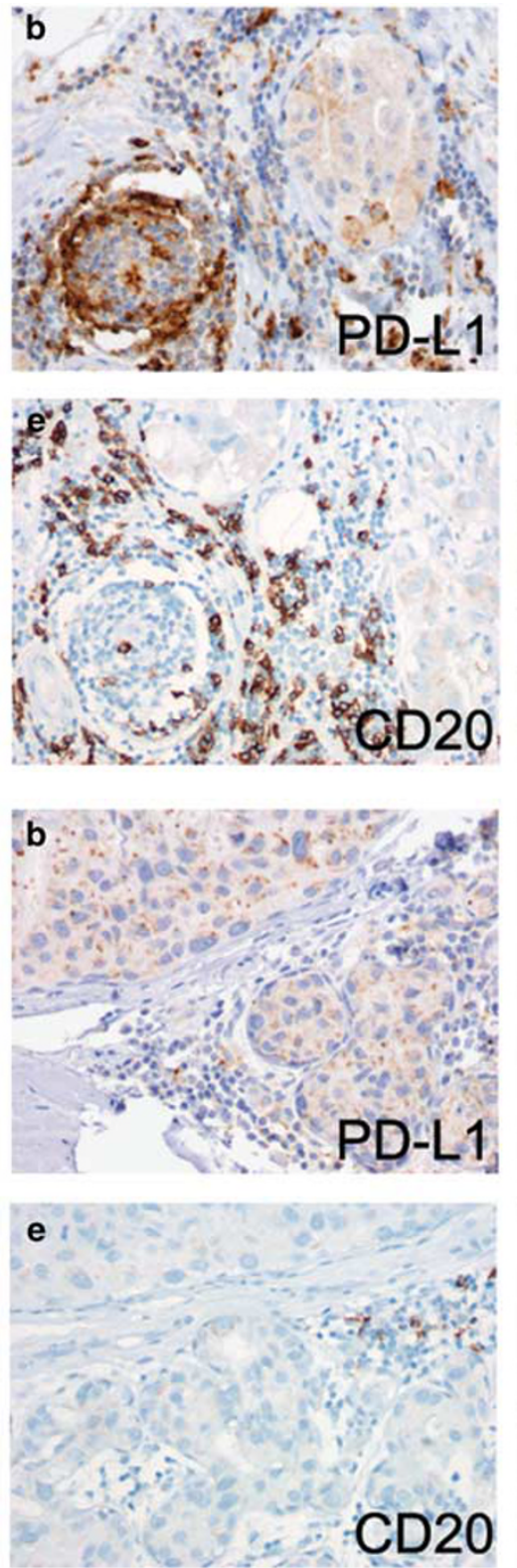
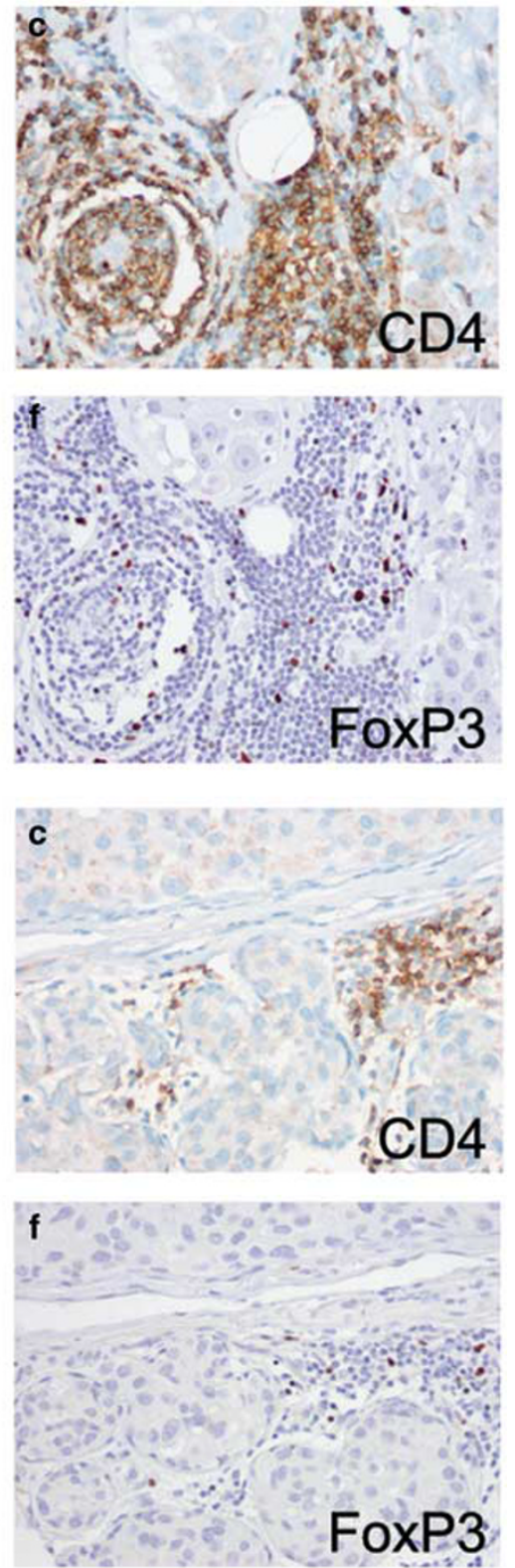

Figure 2 Immunologic features of the ductal carcinoma in situ (DCIS) tumor microenvironment. Most DCIS cases (81\%) (panel 1; a, hematoxylin and eosin, H\&E) display programmed death ligand 1 (PD-L1) tumor infiltrating lymphocytes (b). PD-L1 ${ }^{+}$tumor infiltrating lymphocytes are associated with greater numbers of all tumor infiltrating lymphocyte subsets including CD4 helper T cells (c), CD8 ${ }^{+}$ cytotoxic T cells (d), CD20 ${ }^{+} \mathrm{B}$ cells (e), and FoxP3 ${ }^{+}$Tregs (f) relative to DCIS with PD-L1 ${ }^{-}$tumor infiltrating lymphocytes (panel 2). Importantly, no DCIS carcinoma cell displayed cell surface PD-L1 staining.

infiltrating lymphocytes in ER ${ }^{-}$tumors, ${ }^{11}$ higher levels of $\mathrm{FoxP}^{+}$Tregs in $\mathrm{ER}^{-}$tumors, ${ }^{13}$ strong expression of PD-L1 in ER ${ }^{-}$tumors and triple-negative breast carcinomas, ${ }^{11}$ and tumor infiltrating lymphocyte PD-L1 expression when higher overall levels of tumor infiltrating lymphocytes are present. ${ }^{21}$ The two cases of DCIS that eventually recurred had large numbers of tumor infiltrating lymphocytes, but high levels of $\mathrm{FoxP}^{+}$Treg. Although the conclusions are limited by the small number of recurrences in this series, our data are consistent with prior reports that increased numbers of $\mathrm{FoxP}^{+}$Tregs in DCIS are associated with increased recurrence risk. ${ }^{10}$ These data together suggest that even very early immune responses to breast DCIS may predict future disease behavior.

Our work also demonstrates that while DCIS tumor cells lack PD-L1 expression, the majority of tumor infiltrating lymphocytes associated with DCIS do express PD-L1. We have previously shown that the majority of tumor infiltrating lymphocytes associated with infiltrating ductal carcinoma also express PD-L1, ${ }^{24}$ but that $21 \%$ primary infiltrating ductal carcinoma have PD-L1 ${ }^{+}$carcinoma cells. This dichotomy suggests there may be differences between the character of the immune response to in situ and invasive disease. Little is known 
about the evolution of the immune response and immunologic alterations within the tumor microenvironment along the continuum from in situ to invasive disease. It is not clear whether DCIS eventually develops expression of PD-L1 and gives rise to $\mathrm{PD}-\mathrm{L}^{+}{ }^{+}$invasive carcinoma, or if, alternatively, the presence of an invasive PD-L1 ${ }^{+}$ tumor modulates the immune response within DCIS, leading to expression of PD-L1 by the DCIS tumor cells. If the infiltrating component does not generate a robust enough immune response, then insufficient IFN- $\gamma$ may be generated to upregulate PD-L1 on the surface of DCIS cells. Studies are needed to elucidate the mechanisms behind the lack of PD-L1 expression by pure DCIS and the expression of PD-L1 by invasive tumors.

In addition, there is limited data on the spatial distribution of the immune response within either DCIS or invasive ductal carcinoma. In other tumor types, PD-L1 expression is often seen expressed primarily at the 'leading edge' of the tumor or at the tumor-stromal interface. ${ }^{19}$ This supports the possibility that interactions between invasive tumors and the stromal/immune environment drive PD-L1 expression on tumor cells, which could then modulate the immune milieu of associated in situ lesions. In studies of melanoma, IFN- $\gamma$ and other cytokines were shown to colocalize with tumor cells in PD-L1 ${ }^{+}$tumors, but not in PD-L1 ${ }^{-}$tumors. ${ }^{19,32}$ Such spatial distribution of inflammatory mediators could also contribute to differences in PD-L1 expression in DCIS and invasive breast carcinoma. Indeed, pure DCIS lesions are architecturally distinct from most invasive carcinomas because, while some DCIS are mass-forming lesions that spatially have a 'leading edge,' the majority of DCIS spread diffusely, segmentally, or irregularly through the breast ducts and lack a distinct 'leading edge.'

The expression of immune checkpoint molecules in DCIS identifies them as potential targets for DCIS therapy and secondary breast cancer prevention. DCIS can be responsive to immune-based therapy, ${ }^{30,31,33}$ and PD-L1 antagonists are active in triple-negative breast carcinomas. ${ }^{34,35}$ Although PD-L1 is not expressed on the surface of DCIS tumor cells in pure in situ lesions, the expression of PD-L1 by DCIS tumor infiltrating lymphocytes suggests that targeting PD-L1 is worth investigating as a treatment strategy for high-risk DCIS. ER ${ }^{-}$and HER-2 ${ }^{+}$DCIS and DCIS in young women have the greatest numbers of tumor infiltrating lymphocytes. These patients may be ideal for testing immune-based breast cancer prevention strategies, and this may be particularly true for young women who have a higher DCIS CD8/FoxP3 ratio.

Additionally, we examine tumor infiltrating lymphocyte subsets and PD-L1 expression in DCIS classified into subtypes by ER, PR, and HER-2 status. Our study is limited by the small sample size and lack of nuclear grade 1 cases. In addition, the tissue microarray methodology limits analysis of the geography of tumor infiltrating lymphocytes and PD-L1 expression, including the 'leading edge' of tumors known to be immunologically important in invasive carcinomas. ${ }^{36}$ However, 2-5 cores per tumor were taken to mitigate sampling limitations. Moreover, DCIS also tends to be more diffuse or segmental than invasive breast cancers, which tend to be mass forming, potentially limiting the importance of the 'leading edge' in purely in situ lesions. Finally, the tissue microarray methodology also limits our ability to detect lymphoid aggregates, which have been shown to be important in invasive breast carcinomas. ${ }^{2}$

In conclusion, we demonstrate differential patterns of tumor infiltrating lymphocyte infiltrates in distinct phenotypes of DCIS. Differences are seen relative to ER expression and patient age. Additional studies are needed to further characterize the association of immune parameters with the presence of concurrent infiltrating ductal carcinoma and eventual recurrence in DCIS. We previously reported differential patterns of tumor infiltrating lymphocytes across matched primary and metastatic breast carcinomas. ${ }^{23}$ Our findings in breast DCIS are similar to patterns of tumor infiltrating lymphocytes in primary breast carcinomas, and distinct from patterns of tumor infiltrating lymphocytes in metastatic breast carcinomas. The presence of tumor infiltrating lymphocytes in DCIS, combined with the expression of PD-L1 on tumor infiltrating lymphocytes within the DCIS microenvironment, suggests the potential for immune-based therapies to treat DCIS. Further characterization of the DCIS immune microenvironment may yield additional targets for immune-based therapy and prevention of recurrence, and may help elucidate the role of the immune response in the evolution of breast cancer from in situ to invasive and ultimately to recurrent or metastatic disease.

\section{Disclosure/conflict of interest}

Cimino-Mathews and Emens receive research funding from Genentech and Roche; and Emens receives research funding from EMD Serono, Merck, and Astrazeneca. Taube receives research funding from and is a member of the advisory board for Bristol-Myers Squibb.

\section{References}

1 Emens LA. Breast cancer immunobiology driving immunotherapy: vaccines and immune checkpoint blockade. Expert Rev Anticancer Ther 2012;12:1597-1611.

2 Cimino-Mathews A, Foote JB, Emens LA. Immune targeting in breast cancer. Oncology (Williston Park) 2015;29:375-385.

3 Loi S, Sirtaine N, Piette F et al. Prognostic and predictive value of tumor-infiltrating lymphocytes in a phase III randomized adjuvant breast cancer trial in 
node-positive breast cancer comparing the addition of docetaxel to doxorubicin with doxorubicin-based chemotherapy: BIG 02-98. J Clin Oncol 2013;31:860-867.

4 Adams S, Gray RJ, Demaria S et al. Prognostic value of tumor-infiltrating lymphocytes in triple-negative breast cancers from two phase III randomized adjuvant breast cancer trials: ECOG 2197 and ECOG 1199. J Clin Oncol 2014;32:2959-2967.

5 Loi S, Michiels S, Salgado R et al. Tumor infiltrating lymphocytes are prognostic in triple negative breast cancer and predictive for trastuzumab benefit in early breast cancer: results from the FinHER trial. Ann Oncol 2014;25:1544-1550.

6 Kreike B, van Kouwenhove M, Horlings $\mathrm{H}$ et al. Gene expression profiling and histopathological characterization of triple-negative/basal-like breast carcinomas. Breast Cancer Res 2007;9:R65.

7 Bates GJ, Fox SB, Han C et al. Quantification of regulatory $\mathrm{T}$ cells enables the identification of highrisk breast cancer patients and those at risk of late relapse. J Clin Oncol 2006;24:5373-5380.

8 Perou CM, Sorlie T, Eisen MB et al. Molecular portraits of human breast tumours. Nature 2000;406:747-752.

9 Norum JH, Andersen K, Sorlie T. Lessons learned from the intrinsic subtypes of breast cancer in the quest for precision therapy. Br J Surg 2014;101:925-938.

10 Vincent-Salomon A, Lucchesi C, Gruel N et al. Integrated genomic and transcriptomic analysis of ductal carcinoma in situ of the breast. Clin Cancer Res 2008;14:1956-1965.

11 Schalper KA, Velcheti V, Carvajal D et al. In situ tumor PD-L1 mRNA expression is associated with increased TILs and better outcome in breast carcinomas. Clin Cancer Res 2014;20:2773-2782.

12 Desmedt C, Haibe-Kains B, Wirapati P et al. Biological processes associated with breast cancer clinical outcome depend on the molecular subtypes. Clin Cancer Res 2008;14:5158-5165.

13 Ghebeh H, Barhoush E, Tulbah et al. FOXP3+ Tregs and B7-H1+/PD-1+ T lymphocytes co-infiltrate the tumor tissues of high-risk breast cancer patients: Implication for immunotherapy. BMC Cancer 2008;8:57.

14 Kristensen VN, Vaske CJ, Ursini-Siegel J et al. Integrated molecular profiles of invasive breast tumors and ductal carcinoma in situ (DCIS) reveal differential vascular and interleukin signaling. Proc Natl Acad Sci USA 2012;109:2802-2807.

15 Dong H, Strome SE, Salomao DR et al. Tumorassociated B7-H1 promotes T-cell apoptosis: a potential mechanism of immune evasion. Nat Med 2002;8: 793-800.

16 Butte MJ, Keir ME, Phamduy TB et al. Programmed death-1 ligand 1 interacts specifically with the B7-1 costimulatory molecule to inhibit $\mathrm{T}$ cell responses. Immunity 2007;27:111-122.

17 Mittendorf EA, Philips AV, Meric-Bernstam $\mathrm{F}$ et al. PD-L1 expression in triple-negative breast cancer. Cancer Immunol Res 2014;2:361-370.

18 Gatalica Z, Snyder C, Maney T et al. Programmed cell death 1 (PD-1) and its ligand (PD-L1) in common cancers and their correlation with molecular cancer type. Cancer Epidemiol Biomarkers Prev 2014;23:2965-2970.

19 Taube JM, Anders RA, Young GD et al. Colocalization of inflammatory response with B7-h1 expression in human melanocytic lesions supports an adaptive resistance mechanism of immune escape. Sci Transl Med 2012;4:127ra37.
20 Ghebeh H, Mohammed S, Al-Omair et al. The B7-H1 (PD-L1) T lymphocyte-inhibitory molecule is expressed in breast cancer patients with infiltrating ductal carcinoma: correlation with important high-risk prognostic factors. Neoplasia 2006;8:190-198.

21 Wimberly $\mathrm{H}$, Brown JR, Schalper KA et al. PD-L1 expression correlates with tumor-infiltrating lymphocytes and response to neoadjuvant chemotherapy in breast cancer. Cancer Immunol Res 3: 326-3322014.

22 Muenst S, Schaerli AR, Gao F et al. Expression of programmed death ligand 1 (PD-L1) is associated with poor prognosis in human breast cancer. Breast Cancer Res Treat 2014;146:15-24.

23 Cimino-Mathews A, Ye X, Meeker A et al. Metastatic triple-negative breast cancers at first relapse have fewer tumor-infiltrating lymphocytes than their matched primary breast tumors: a pilot study. Hum Pathol 2013;44:2055-2063.

24 Cimino-Mathews A, Thompson E, Taube JM et al. PD-L1 (B7-H1) expression and the immune tumor microenvironment in human primary and metastatic breast carcinomas. Hum Pathol 2016;47:52-63.

25 VandenBussche CJ, Elwood H, Cimino-Mathews et al. Clinicopathologic features of ductal carcinoma in situ in young women with an emphasis on molecular subtype. Hum Pathol 2013;44:2487-2493.

26 Cimino-Mathews A, Subhawong AP, Elwood $\mathrm{H}$ et al. Neural crest transcription factor Sox10 is preferentially expressed in triple-negative and metaplastic breast carcinomas. Hum Pathol 2013;44:959-965.

27 Salgado R, Denkert C, Demaria S et al. The evaluation of tumor-infiltrating lymphocytes (TILs) in breast cancer: recommendations by an International TILs Working Group 2014. Ann Oncol 2015;26:259-271.

28 Ursini-Siegel J, Cory S, Zuo D et al. Receptor tyrosine kinase signaling favors a protumorigenic state in breast cancer cells by inhibiting the adaptive immune response. Cancer Res 2010;70:7776-7787.

29 Lee AH, Happerfield LC, Bobrow LG et al. Angiogenesis and inflammation in ductal carcinoma in situ of the breast. J Pathol 1997;181:200-206.

30 Fracol M, Xu S, Mick R et al. Response to HER-2 pulsed DC1 vaccines is predicted by both HER-2 and estrogen receptor expression in DCIS. Ann Surg Oncol 2013;20: 3233-3239.

31 Sharma A, Koldovsky U, Xu S et al. HER-2 pulsed dendritic cell vaccine can eliminate HER-2 expression and impact ductal carcinoma in situ. Cancer 2012;118: 4354-4362.

32 Taube JM, Young GD, McMiller TL et al. Differential expression of immune-regulatory genes associated with PD-L1 display in melanoma: implications for PD-1 pathway blockade. Clin Cancer Res 2015;21:3969-3976.

33 Czerniecki BJ, Roses RE, Koski GK. Development of vaccines for high-risk ductal carcinoma in situ of the breast. Cancer Res 2007;67:6531-6534.

34 Emens LA BF, Cassier P, DeLord JP et al. Inhibition of PD-L1 by MPDL3280A leads to clinical activity in patients with metastatic triple-negative breast cancer. Cancer Res 2015;75:PD1-PD6.

35 Lipson EJ, Forde PM, Hammers HJ et al. Antagonists of PD-1 and PD-L1 in cancer treatment. Semin Oncol 2015;42:587-600.

36 Quail DF, Joyce JA. Microenvironmental regulation of tumor progression and metastasis. Nat Med 2013;19: 1423-1437. 